\section{Commentary: Total lung sparing for low-grade bronchial malignancies: Technically feasible, but still needs long-term survival data}

\author{
Yang Zhang, MD, and Haiquan Chen, MD, PhD
}

Primary bronchial malignancies are relatively rare and include a variety of pathologic types. An important component of minimally invasive thoracic surgery is to remove the tumors completely while also spare normal lung tissue as much as possible. ${ }^{1}$ This is especially the case with lowgrade bronchial malignancies that require minimal resection margins to be curative. In 1955, Paulson and Shaw ${ }^{2}$ made the first significant report on the clinical application of lung-sparing bronchial sleeve resections. Since then, it has become an attractive technique for patients with low-grade bronchial malignancies.

Chen and colleagues ${ }^{3}$ performed a retrospective analysis of 30 patients who underwent left secondary carinal resection and reconstruction with pulmonary parenchymal preservation for low-grade bronchial malignancies, including adenoid cystic carcinoma, mucoepidermoid carcinoma, and carcinoid tumors. The authors present impressive short-term outcomes. There was no anastomotic defect or operative mortality. Only 4 patients had postoperative complications that were successfully handled. Given the rarity of this technically demanding procedure, this study represents a large series. The pictures and video of surgical

From the Departments of Thoracic Surgery and State Key Laboratory of Genetic Engineering, Fudan University Shanghai Cancer Center, Shanghai, China; Institute of Thoracic Oncology, Fudan University, Shanghai, China; and Department of Oncology, Shanghai Medical College, Fudan University, Shanghai, China.

Disclosures: The authors reported no conflicts of interest.

The Journal policy requires editors and reviewers to disclose conflicts of interest and to decline handling or reviewing manuscripts for which they may have a conflict of interest. The editors and reviewers of this article have no conflicts of interest.

Received for publication May 31, 2021; revisions received May 31, 2021; accepted for publication June 5, 2021; available ahead of print June 10, 2021.

Address for reprints: Haiquan Chen, MD, PhD, Department of Thoracic Surgery, Fudan University Shanghai Cancer Center, 270 Dong-An Rd, Shanghai 200032,

China (E-mail: hqchen1@yahoo.com).

JTCVS Techniques 2021;8:202

2666-2507

Copyright (C) 2021 The Author(s). Published by Elsevier Inc. on behalf of The American Association for Thoracic Surgery. This is an open access article under the CC BY-NC-ND license (http://creativecommons.org/licenses/by-nc-nd/4.0/).

https://doi.org/10.1016/j.xjtc.2021.06.011

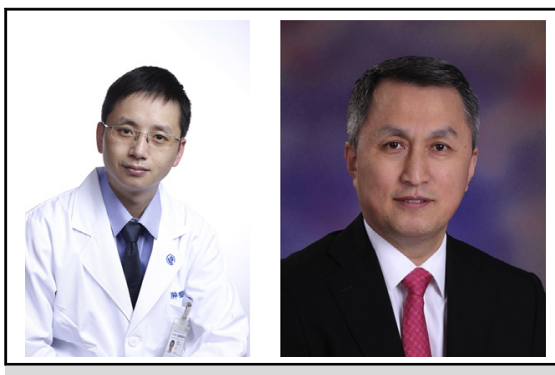

Yang Zhang, MD, and Haiquan Chen, MD, PhD

CENTRAL MESSAGE

Left secondary carinal resection and reconstruction for lowgrade bronchial malignancies is technically feasible. Long-term follow-up is still needed to investigate the oncologic therapeutic effect.

procedures are well done, and can provide useful technical guidance.

Nevertheless, several issues need to be considered when interpreting these results. First, the indications for this procedure need to be more specific. Patients in this study were generally very young, with a median age of 39 years. This partly contributes to the excellent postoperative outcomes. Second, there is a lack of control group. Other treatment modalities, such as endoscopic treatment may also be suitable for selected patients with low-grade bronchial malignancies. Last and most importantly, we still need long-term survival data to further justify its application. The resected margins were positive in $27 \%$ of patients, which might jeopardize long-term survival.

This study demonstrates left secondary carinal resection and reconstruction for selected patients with low-grade bronchial malignancies is technically feasible. Long-term follow-up is still needed to investigate the oncologic therapeutic effect.

\section{References}

1. Cheng X, Onaitis MW, D'Amico TA, Chen H. Minimally invasive thoracic surgery 3.0: lessons learned from the history of lung cancer surgery. Ann Surg. 2018;267: 37-8.

2. Paulson DL, Shaw RR. Preservation of lung tissue by means of bronchoplastic procedures. Am J Surg. 1955;89:347-55.

3. Chen L, Campisi A, Wang Z, Amore AD, Ciarrocchi AP, Zhao H, et al. Left secondary carinal resection and reconstruction for low-grade bronchial malignancies. J Thorac Cardiovasc Surg Tech. 2021;8:196-201. 